\title{
Omalgia crónica e associação com incapacidade funcional e ansiedade/depressão: a realidade de cinco Unidades de Saúde Familiar da Área Metropolitana do Porto
}

Ana Catarina Sá Machado, ${ }^{1}$ Cláudio Sousa Martins, ${ }^{2}$ Joana M. Ferreira, ${ }^{3}$ Rita Sampaio Santos, ${ }^{4}$ Sofia Marçalo, ${ }^{5}$ Sofia Vale ${ }^{6}$

\section{RESUMO}

Objetivos: Estimar a prevalência da omalgia crónica na população de cinco Unidades de Saúde Familiar (USF), descrever as suas características semiológicas e as características sociodemográficas desta população. Secundariamente pretende-se avaliar e quantificar a associação entre omalgia crónica e incapacidade funcional, características sociodemográficas e a perturbação ansiosa/depressiva.

Tipo de estudo: Observacional, transversal, analítico.

Local: Cinco USF da Área Metropolitana do Porto.

População: Calculou-se uma amostra de 1.718 utentes entre os 18 e 64 anos, que se deslocaram às cinco USF durante o estudo. Métodos: Aplicou-se um questionário de recolha de variáveis sociodemográficas e sobre omalgia crónica e duas escalas validadas para a população Portuguesa: Health Assessment Questionnaire-Disability Index e Hospital Anxiety and Depression Scale. Testou-se a associação entre omalgia crónica, incapacidade funcional e ansiedade/depressão através do modelo de regressão logística multinomial. Resultados: A prevalência estimada de omalgia crónica foi de $29,6 \%$, intervalo de confiança de $95 \%$ [27,4-31,8]. Em 57,8\% dos utentes a dor era diária, 70,7\% tinha dor moderada a intensa e 41,1\% encontrava-se pouco ou nada satisfeito com o tratamento. Verificou-se uma associação entre a existência e localização da dor com o estado emocional, género, estado civil, escolaridade, reforma e reforma por invalidez $(p<0,001)$. Nos indivíduos com omalgia crónica, as mulheres, divorciados e viúvos, com baixa escolaridade, reformados por invalidez e com ansiedade/depressão apresentaram uma proporção superior. Constatou-se a existência de um aumento do odds ratio (OR) de omalgia crónica com o aumento do grau de incapacidade, da ansiedade, da idade e com a baixa escolaridade (inferior ou igual a nove anos).

Conclusões: Apesar de prevista uma prevalência de omalgia crónica em Portugal de 4,4\% com base em dados bibliográficos, a prevalência encontrada neste estudo foi aproximadamente sete vezes superior à esperada. Verificou-se uma associação da omalgia crónica com o grau de incapacidade e a ansiedade, o que vai de encontro ao debatido na literatura. O presente estudo mostra uma necessidade urgente de intervir eficazmente no controlo da omalgia crónica.

Palavras-chave: Omalgia crónica; Incapacidade funcional; Ansiedade; Depressão.

\section{INTRODUÇÃO}

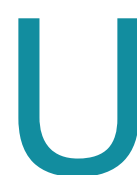

ma das definições de dor crónica mais utilizada é "dor com duração superior a três meses ou que persiste após o período normal de recuperação do tecido, na ausência de outros critérios".
1. UCSP Armamar, ACeS Douro Sul.

2. USF Arco do Prado, ACeS Gaia.

3. USF Azevedo Campanhã, ACeS Porto Oriental.

4. USF Lusa, ACeS Lisboa Ocidental - Oeiras.

5. USF S. Félix/Perosinho, ACeS Espinho/Gaia.

6. USF Fonte de Água, AceS Cascais. 
Cerca de 36,7\% da população adulta Portuguesa sofre de dor crónica. ${ }^{2}$ Estima-se que entre esta população, $92 \%$ reporte algum grau de incapacidade funcional associada. ${ }^{2}$ Os doentes com dor crónica também desenvolvem facilmente sintomas psicopatológicos reativos, principalmente de ansiedade e depressão, quer pela intensidade quer pela interferência da dor no seu quotidiano. ${ }^{3} \mathrm{O}$ estudo de Azevedo e colaboradores reportou uma prevalência de $25 \%$ de sintomatologia depressiva e de $29 \%$ de sintomas de ansiedade na população com dor crónica. ${ }^{2}$

Cerca de $12 \%$ da população adulta Portuguesa que sofre de dor crónica localiza a dor a nível do ombro. ${ }^{2} \mathrm{~A}$ omalgia pode resultar maioritariamente de lesões degenerativas ou traumáticas que podem acometer as estruturas moles ou a articulação do ombro, podendo também corresponder a uma dor irradiada ou referida. ${ }^{4-5}$

A presença de dor e rigidez no ombro pode levar a uma incapacidade para o trabalho e/ou para as atividades domésticas e recreativas, criando assim uma elevada carga de doença, tanto para o indivíduo como para a sociedade. ${ }^{6}$ A omalgia constitui uma causa frequente de consulta nos cuidados de saúde primários (CSP), bem como de referenciação para tratamentos de medicina física e de reabilitação, acarretando gastos significativos em cuidados de saúde. ${ }^{4,7}$ Apesar de a omalgia crónica ser um problema comum nos CSP, não existem estudos em Portugal que traduzam a sua prevalência dissociada da prevalência da dor crónica nem a prevalência no âmbito dos CSP. Os estudos existentes são referentes à dor crónica em geral, sendo que as conclusões obtidas são generalizadas e comuns à dor crónica em várias localizações, não se percebendo o real impacto e contribuição da omalgia crónica, enquanto fator isolado, nos resultados desses estudos.

O presente estudo teve como objetivos primários estimar a prevalência da omalgia crónica na população de cinco Unidades de Saúde Familiar (USF) da Área Metropolitana do Porto, descrever as características sociodemográficas da população com omalgia crónica e as características semiológicas da omalgia crónica nesta população. Como objetivos secundários pretendeuse avaliar e quantificar a associação entre omalgia crónica e incapacidade funcional, características sociodemográficas e a perturbação ansiosa/depressiva.

\section{MÉTODOS}

\section{Desenho do estudo}

Realizou-se um estudo observacional, transversal e analítico a partir de uma amostra de conveniência da população de utentes, inscritos e esporádicos, que se deslocou às cinco USF durante o período de estudo. Foi aplicado um questionário composto por três partes, de forma a avaliar a omalgia crónica, a incapacidade funcional e a existência de ansiedade/depressão.

A primeira parte do questionário consistiu num formulário, construído pelos investigadores, que incluiu questões sobre características sociodemográficas da população:

1. Sexo (masculino; feminino) e idade.

2. Estado civil (solteiro; casado/união de facto; divorciado/separado; viúvo).

3 . Escolaridade $\left(1 .^{\circ}\right.$ ciclo; $2 .^{\circ}$ e $3 .^{\circ}$ ciclo; ensino secundário; ensino superior).

4. Situação laboral atual (ativo; reformado; reformado por invalidez).

Esta parte do questionário incluiu ainda questões sobre características semiológicas da omalgia crónica:

1. Presença de dor crónica [definição da International Association for the Study of Pain (IASP)]. ${ }^{8}$

2. Presença de omalgia crónica.

3. Duração da omalgia crónica (menos de um ano; um a dois anos; três a cinco anos; seis a dez anos; mais de dez anos).

4. Frequência da omalgia crónica (uma vez ou menos por mês; duas ou mais vezes por mês; uma ou mais vezes por semana; uma ou mais vezes por dia; sempre).

5. Intensidade da omalgia crónica (escala numérica da dor). ${ }^{9}$

6. Conduta terapêutica [nada; autocuidados (aplicação de calor ou frio, alongamentos, massagem em casa, correção da postura); automedicação (medicamentos não prescritos pelo médico); medicamentos prescritos pelo médico; medicinas alternativas (massagem, acupunctura, produtos da ervanária, osteopatia, homeopatia, etc.); outros].

7. Satisfação com o tratamento (nada satisfeito; pouco satisfeito; moderadamente satisfeito; satisfeito; totalmente satisfeito).

Na segunda parte do questionário foi avaliada a capacidade funcional através do Health Assessment 
Questionnaire-Disability Index (HAQ-DI), escala validada para a população portuguesa. ${ }^{10-13}$ Este questionário é constituído por 20 perguntas que avaliam oito categorias de atividades: vestir-se, levantar-se, comer, deambular, higiene pessoal, alcançar, agarrar e outras atividades habituais. Para cada item há uma escala de dificuldade de quatro níveis que é pontuada de zero a três, representando 0 - sem dificuldade/normal, 1 - alguma dificuldade, 2 - muita dificuldade e 3 - incapacidade. A pontuação global da HAQ-DI é a média das oito categorias numa escala de zero (normal) a três (incapacidade grave).

A terceira parte do questionário consistiu na Escala Hospitalar de Depressão e Ansiedade (Hospital Anxiety and Depression Scale-HADS), também validada para a população portuguesa. ${ }^{14-16}$ Esta escala está dividida em duas subescalas, uma que mede a ansiedade (HADS-A), com sete itens, e outra que mede a depressão (HADS-D), também com sete itens, que são pontuados separadamente. Cada item é respondido pelo utente com uma de quatro opções possíveis, pontuadas individualmente de zero a três. O significado atribuído às pontuações em ambas as subescalas é o seguinte: HADS-A - com ansiedade $\geq 11$ pontos; HADS-D - com depressão $\geq 11$ pontos.

O questionário foi testado em 10 utentes antes da sua aplicação.

\section{Contexto, população do estudo e tamanho amostral}

A população do estudo englobou os utentes inscritos e esporádicos, com idades compreendidas entre os 18 e os 64 anos, que se deslocaram a cinco USF de quatro Agrupamentos de Centros de Saúde (ACeS) da Área Metropolitana do Porto $(n=47.289)$ : ACeS Grande Porto II - USF Valbom; ACeS Grande Porto III - USF Saúde em Família; ACeS Grande Porto VII - USF Arco do Prado e USF Nova Salus; ACeS Grande Porto VIII - USF S. Félix/Perosinho.

De acordo com a bibliografia estima-se que a prevalência de dor crónica em Portugal seja de $36,7 \%$ e que a prevalência de omalgia nos que sofrem de dor crónica seja de $12 \%{ }^{2}$ Relativamente ao cálculo do tamanho amostral e com base nas percentagens encontradas na bibliografia, porque não existe um valor real da omalgia crónica em Portugal, os autores realizaram um cálculo aproximado estimando uma prevalência de omalgia crónica em Portugal de 4,4\%.
Considerando a ausência de evidência relativa à prevalência da omalgia crónica, e sabendo que entre os doentes com dor crónica $92 \%$ reporta algum grau de incapacidade, ${ }^{2}$ estima-se que a prevalência de omalgia crónica com incapacidade funcional na população geral seja de $4,05 \%$. Da mesma forma, sabe-se que a prevalência de sintomas depressivos e ansiosos na população com dor crónica em Portugal corresponde a 25\% e $29 \%$, respetivamente, ${ }^{2}$ pelo que a prevalência esperada é de $1,1 \%$ e $1,28 \%$ neste estudo.

O tamanho da amostra foi calculado considerando um intervalo de confiança (IC) de 95\% e um grau de precisão de $1 \%$, o que correspondeu a um total de 1.562 utentes (cálculo efetuado no programa EpiInfo $7^{\circledR}$ ). Assumindo $10 \%$ de questionários anulados por missing data, pretendeu-se uma amostra de conveniência de 1.718 utentes. Na Tabela 1 apresenta-se a distribuição do número de questionários por USF.

Foram excluídos os utentes com incapacidade para responder de forma autónoma ao questionário de investigação.

\section{Aplicação dos questionários}

Os utentes, em cada USF, foram convidados a participar no estudo quando se dirigiram ao secretariado da mesma. Os questionários estiveram disponíveis nas USF entre os meses de setembro e dezembro de 2017. Por forma a evitar a duplicação de respostas aos questionários, os utentes, aquando do convite para o seu preenchimento, eram interpelados pelos secretários clínicos acerca do preenchimento prévio do mesmo, sendo que, em caso afirmativo, não lhes era facultado novo questionário. Nos locais estiveram também disponíveis caixas para colocar os questionários preenchidos, de forma a garantir a confidencialidade e anonimato. Os questionários eram recolhidos a cada sete dias por um investigador externo à unidade.

\section{Análise estatística}

Foi realizada uma análise descritiva das variáveis, através da apresentação das frequências e proporções para as variáveis categóricas e da média e desvios-padrão para as variáveis contínuas.

Foram considerados critérios de exclusão: ausência de identificação da idade; ausência de resposta relativa 


TABELA 1. Distribuição do número de questionários por Unidade de Saúde Familiar
\begin{tabular}{l|c|c|c} 
USF & População elegível & Proporção da amostra & N. ${ }^{\circ}$ questionários \\
\hline USF Valbom & 8.571 & $18,12 \%$ & 311 \\
\hline USF Saúde em Família & 8.918 & $18,86 \%$ & 324 \\
\hline USF Arco do Prado & 8.153 & $17,24 \%$ & 296 \\
\hline USF Nova Salus & 8.773 & $18,55 \%$ & 319 \\
\hline USF S. Félix/Perosinho & 12.874 & $27,22 \%$ & 468 \\
\hline Total & 47.289 & $100 \%$ & 1.718 \\
\hline
\end{tabular}

\section{RESULTADOS}

Foram respondidos um total 1.697 questionários, dos quais foram excluídos 104 $(6,1 \%)$ atendendo aos critérios de exclusão. Deste modo, participaram no estudo um total de 1.593 indivíduos.

No total da amostra, a prevalência de dor crónica obtida foi de $45,3 \%$ ( $n=721$ ), sendo a prevalência de omalgia

à presença de dor há pelo menos três meses e/ou de dor localizada ao ombro; ausência de resposta e/ou resposta múltipla (exceto na pergunta relacionada com a conduta terapêutica) em duas ou mais questões no formulário construído pelos investigadores; ausência de resposta em três ou mais categorias de atividades da HAQ-DI; e ausência de resposta em dois ou mais itens de cada uma das subescalas da HADS.

Realizou-se uma análise bivariada dos dados através do teste do qui-quadrado para as variáveis categóricas, para avaliar a associação das variáveis sociodemográficas (sexo, idade, estado civil, escolaridade e situação laboral atual) e a presença de omalgia crónica. Utilizou-se o teste ANOVA para analisar as variáveis contínuas referentes às pontuações da HAQ-DI e HADS, nomeadamente para avaliar se as médias destas pontuações são significativamente diferentes em cada grupo de dor (dor crónica não localizada ao ombro, omalgia crónica, ausência de dor). O teste $t$ deStudent foi utilizado para testar a hipótese de existência de diferença significativa entre os sexos na pontuação média de incapacidade funcional em indivíduos com omalgia crónica.

Através de uma regressão logística multinomial calcularam-se os OR para a omalgia crónica, em função das variáveis sociodemográficas (sexo, idade, estado civil, escolaridade e situação laboral atual) e da incapacidade funcional e ansiedade/depressão.

Adotou-se um nível de significância de 0,05. Realizou-se a análise de dados utilizando o software Statistical Package for the Social Sciences (SPSS), versão 20.0 ${ }^{\oplus}$.

O protocolo do estudo foi aprovado pelos Conselhos Clínicos de Saúde dos quatro ACeS e pela Comissão de Ética da Administração Regional de Saúde do Norte. naqueles que sofriam de dor crónica de $65,5 \%$ ( $n=472$ ). A prevalência estimada de omalgia crónica na população estudada foi de 29,6\%, IC95\% [27,4-31,8].

Relativamente às características da omalgia, 33,6\% dos indivíduos $(n=158$; total=470) referiu ter omalgia há menos de um ano e $31,4 \%(n=148$; total $=472)$ referiu que a frequência da omalgia era de caráter contínuo (Figura 1). Quanto à intensidade da omalgia crónica obtiveram-se 420 respostas, com uma média de intensidade de 6,5 $\pm 2,1$ (mínimo de zero e máximo de 10). Apenas 5,9\% dos indivíduos referiu ter dor de intensidade inferior ou igual a três, enquanto cerca de $70,7 \%$ referiu dor de intensidade igual ou superior a seis (Figura 2). Ao nível da conduta terapêutica da omalgia, $44,1 \%$ ( $n=208)$ referiu autocuidados, $43,4 \%(n=205)$ medicamentos prescritos pelo médico, $18,2 \%$ ( $n=86$ ) automedicava-se, $14,4 \%$ ( $n=68)$ utilizava medicinas alternativas, $14,4 \%$ ( $n=68$ ) não fazia nada e 8,8\% ( $n=40)$ tomava outras atitudes (e.g., tratamento fisiátrico ou hidroginástica). Cerca de $37 \%$ dos indivíduos reportou ter mais do que uma atitude terapêutica das anteriormente descritas para alívio da dor. Relativamente à satisfação com a conduta terapêutica, 179 indivíduos $(44,1 \%)$ mostraram-se moderadamente satisfeitos (Figura 3 ).

A idade dos participantes variou entre os 18 e os 64 anos (média de $42,7 \pm 12,5$ anos). As restantes características sociodemográficas e os resultados da análise logística multinomial efetuada para testar a associação entre omalgia crónica e incapacidade funcional, variáveis sociodemográficas e depressão/ansiedade encontram-se sumariados na Tabela 2.

No grupo da omalgia crónica, comparativamente aos grupos sem dor e com dor crónica noutra localização, verificou-se que a proporção de mulheres era superior. 


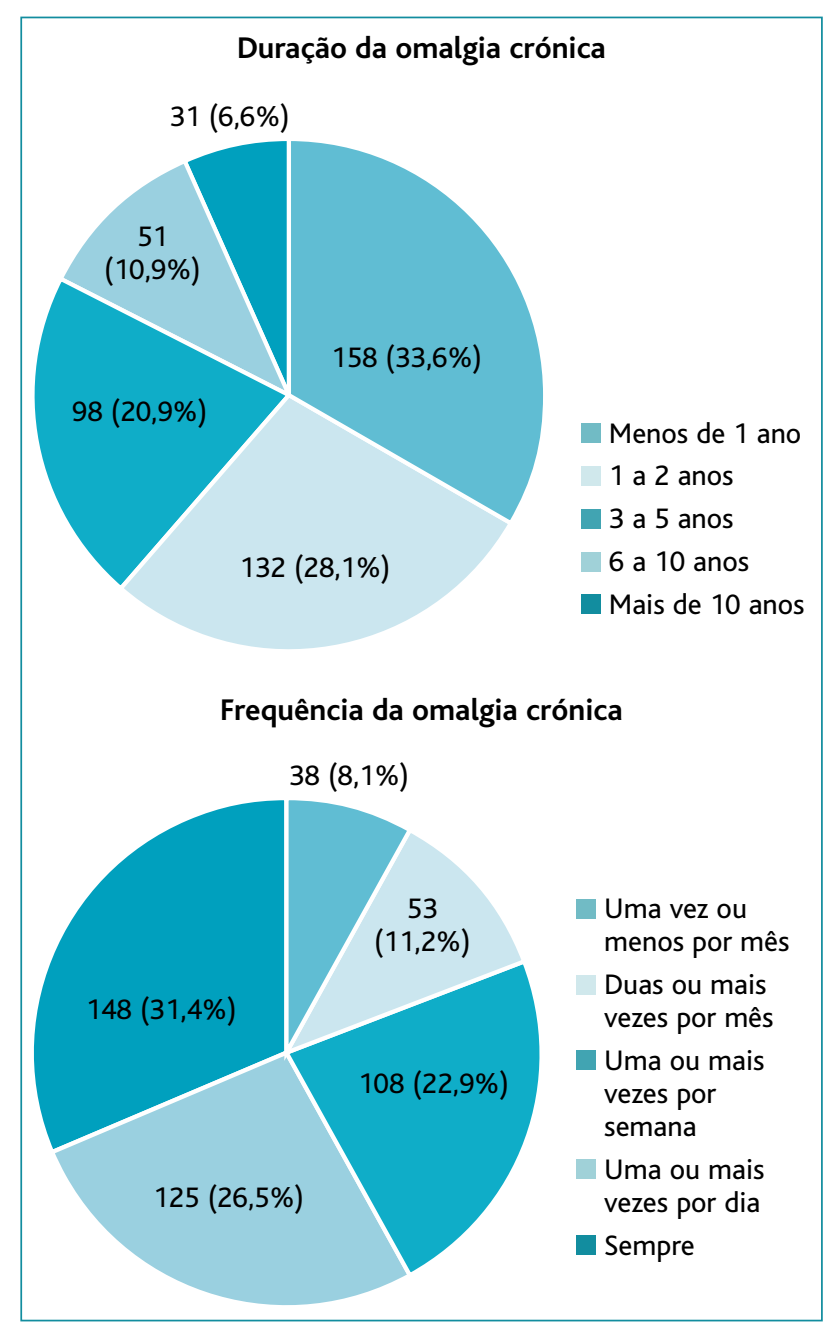

Figura 1. Duração e frequência da omalgia crónica na amostra.

Em relação ao estado civil também havia mais indivíduos divorciados e viúvos ( $n=91$ e $n=19$, respetivamente). Constatou-se ainda que havia maior proporção de indivíduos com escolaridade inferior ou igual a nove anos e maior proporção dos indivíduos reformados por invalidez. Em relação à ansiedade e presença conjunta de ansiedade e depressão verificou-se um valor superior no grupo de indivíduos com omalgia crónica.

Das 1.531 respostas válidas na escala HAQ-DI, 683 indivíduos $(44,6 \%)$ reportaram algum grau de incapacidade, enquanto no grupo da omalgia crónica esta foi reportada em $84,0 \%$.

Através da análise multinomial (Tabela 2) verificou-se que a pontuação média de incapacidade foi signi-

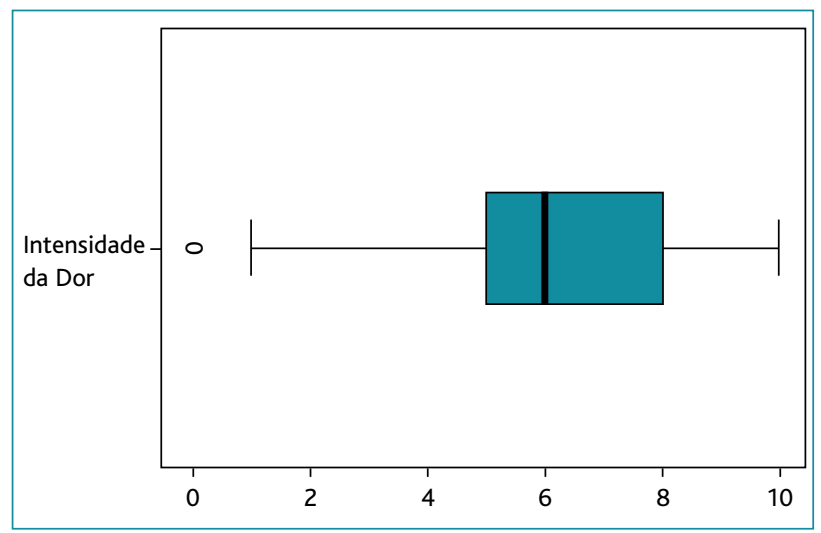

Figura 2. Intensidade da omalgia crónica na amostra.

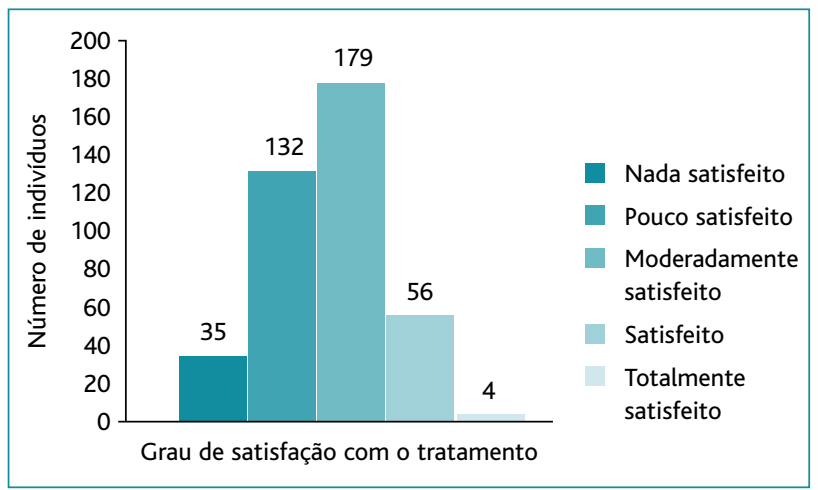

Figura 3. Satisfação com o tratamento da omalgia crónica na amostra.

ficativamente superior nos indivíduos com ansiedade e depressão, género feminino, viúvos e reformados. Não houve associação estatisticamente significativa na escolaridade, situação laboral e reforma por invalidez. Ainda relativamente à associação entre idade e incapacidade funcional, verificou-se que os indivíduos com omalgia crónica eram mais velhos e apresentaram valores médios de incapacidade mais elevados ( $p<0,001$, teste ANOVA) (Tabela 3).

Ajustando a todas as variáveis e no sentido de estabelecer uma associação independente através do cálculo dos OR (Tabela 4), constatou-se que o OR da omalgia crónica aumenta 52,6 vezes por cada unidade de aumento de incapacidade. Para além disso, o OR da omalgia crónica aumenta com a ansiedade (15,0\%), idade $(3,1 \%)$ e baixa escolaridade (zero aos quatro anos e cinco aos nove anos, $161,3 \%$ e $86,9 \%$, respetivamente). 


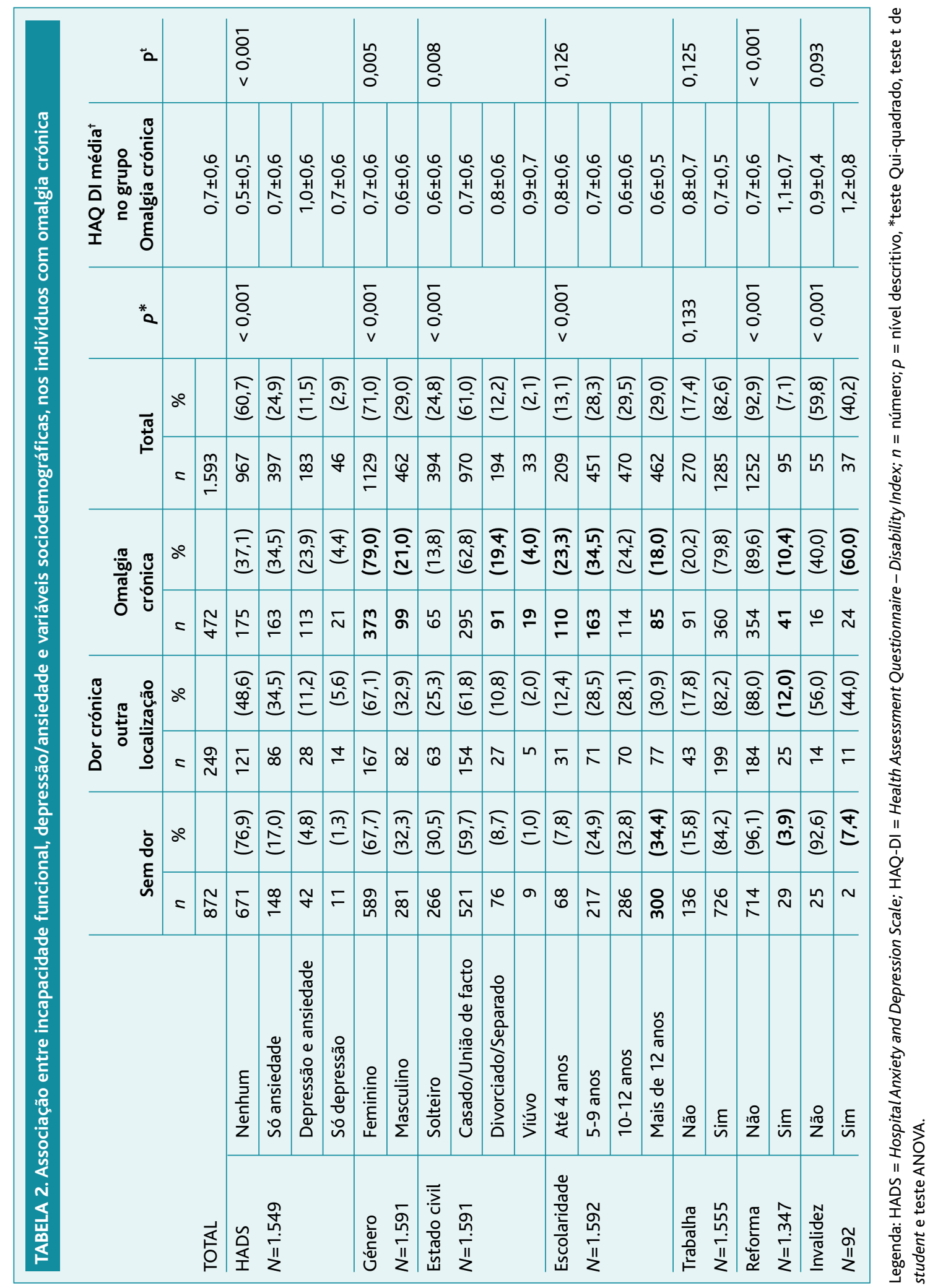


TABELA 3. Associação entre idade e incapacidade funcional nos indivíduos com omalgia crónica

\begin{tabular}{|c|c|c|c|c|c|c|}
\hline & & $n$ & Mínimo & Máximo & Média \pm Desvio-padrão & $p^{*}$ \\
\hline \multirow[t]{3}{*}{ Idade } & Sem dor & 872 & 18 & 64 & $39,3 \pm 12,3$ & $<0,001$ \\
\hline & Dor crónica excluindo omalgia & 249 & 18 & 64 & $44,1 \pm 12,3$ & \\
\hline & Omalgia crónica & 472 & 18 & 64 & $48,1 \pm 10,8$ & \\
\hline \multirow[t]{3}{*}{ Score HAQ-DI } & Sem dor & 872 & 0 & 2 & $0,1 \pm 0,2$ & $<0,001$ \\
\hline & Dor crónica excluindo omalgia & 249 & 0 & 2 & $0,4 \pm 0,4$ & \\
\hline & Omalgia crónica & 472 & 0 & 3 & $0,7 \pm 0,6$ & \\
\hline
\end{tabular}

Legenda: HAQ-DI = Health Assessment Questionnaire Disability Index; $n=$ número; $p$ = nível descritivo, *Teste de ANOVA.

\begin{tabular}{|c|c|c|c|c|}
\hline \multicolumn{2}{|c|}{ Omalgia crónica } & OR & IC $95 \%$ & $P$ \\
\hline \multicolumn{2}{|l|}{ Score HAQ-DI } & 52,594 & $24,732-93,037$ & $<0,001$ \\
\hline \multirow[t]{2}{*}{ HADS } & Total Ansiedade & 1,150 & $1,097-1,205$ & $<0,001$ \\
\hline & Total Depressão & 1,035 & $0,983-1,091$ & 0,192 \\
\hline \multicolumn{2}{|l|}{ Idade } & 1,031 & $1,014-1,047$ & $<0,001$ \\
\hline Género & Feminino & 0,906 & $0,633-1,296$ & 0,588 \\
\hline \multirow[t]{3}{*}{ Estado civil } & Solteiro & 0,351 & $0,097-1,263$ & 0,109 \\
\hline & Casado/União de facto & 0,351 & $0,105-1,180$ & 0,091 \\
\hline & Divorciado/Separado & 0,500 & $0,141-1,773$ & 0,283 \\
\hline \multirow[t]{3}{*}{ Escolaridade } & Até 4 anos & 2,613 & $1,511-4,519$ & 0,001 \\
\hline & $5-9$ anos & 1,869 & $1,228-2,844$ & 0,004 \\
\hline & 10-12 anos & 1,047 & $0,687-1,594$ & 0,832 \\
\hline
\end{tabular}

Legenda: $\mathrm{HAQ}-\mathrm{DI}=$ Health Assessment Questionnaire - Disability Index; $n=$ número; $\mathrm{OR}=$ odds ratio; $p$ = nível descritivo.

\section{DISCUSSÃO}

A epidemiologia da dor crónica está ainda pouco estudada em Portugal. No entanto, através dos estudos nacionais ${ }^{2}$ e europeus ${ }^{17}$ sabe-se que representa um problema de saúde pública importante. A omalgia é referida em alguns estudos de dor crónica, mas até à data não existem trabalhos dirigidos especificamente a esta patologia, estando apenas disponíveis trabalhos que a analisam dentro da problemática da dor crónica, sem que seja possível tirar conclusões específicas acerca do real impacto da omalgia enquanto fator isolado, na associação com incapacidade funcional e ansiedade/
/ depressão. O objetivo deste estudo foi colmatar essa falta de evidência científica, pelo que os autores julgam que se torna um estudo que acrescenta especificidade à evidência científica existente por permitir a análise unitária e singular da omalgia crónica dissociada dos restantes tipos de dor crónica.

Assim, este estudo apresenta vários pontos fortes. Em primeiro lugar, foi desenhado para estudar especificamente a epidemiologia da omalgia crónica isolada e o seu impacto funcional e associação com ansiedade/depressão. Em segundo lugar, foram utilizados métodos rigorosos de amostragem, foi obtida uma amostra significativa e foram usadas definições padronizadas e medidas validadas em cada escala constante no questionário. Em terceiro, trata-se de um estudo dirigido à população frequentadora dos CSP, tendo por isso um âmbito mais específico que outros estudos de dor crónica em Portugal.

No total da amostra estudada obteve-se uma prevalência de dor crónica de 45,3\%, um valor superior ao encontrado no estudo de Azevedo e colaboradores, ${ }^{2}$ realizado em Portugal em 2012, que apresentava $36,7 \%$. Contudo, convém salientar que esse estudo teve em conta, para cálculo do tamanho da amostra, uma prevalência de dor crónica de $20 \%$ que foi inferida de estudos prévios, com uma variação que oscilou entre $2 \mathrm{e}$ $55 \%$ na prevalência. Nesse estudo, dos doentes que 
sofriam de dor crónica, a percentagem daqueles que localizou a dor ao nível do ombro foi de $12 \%,{ }^{2}$ enquanto a percentagem obtida no presente estudo foi de $65,5 \%$. A prevalência de omalgia crónica calculada no presente estudo foi de $29,6 \%$, uma percentagem cerca de sete vezes superior à estimativa efetuada inicialmente $(4,4 \%)$. De ressalvar que a percentagem de omalgia crónica de $4,4 \%$ foi estimada pelos autores a partir das prevalências do estudo já referido, ${ }^{2}$ não tendo sido nele especificamente determinada. Estas diferenças podem justificar-se pelo facto de se estar perante uma amostra de conveniência, podendo-se estar a sobrestimar a prevalência de omalgia crónica. Além disso, também os utentes com omalgia crónica podem ter estado mais motivados para a conclusão do preenchimento do questionário e a sua entrega. Por outro lado, este tipo de amostra não considerou utentes que não procuraram assistência médica durante o período de estudo, o que pode ter potenciado um viés de seleção. Desta forma, as prevalências calculadas neste estudo são referentes à população frequentadora dos CSP, contrariamente às prevalências obtidas no estudo de Azevedo e colaboradores, ${ }^{2}$ que são referentes à população geral e que resultam da análise dos dados obtidos de respostas telefónicas a um questionário, não diferenciando utentes frequentadores de CSP de utentes não frequentadores.

Analisando as características da omalgia, verificou-se que cerca de $57,8 \%$ dos doentes sentem dor diariamente, $70,7 \%$ têm dor de intensidade moderada a intensa e $41,1 \%$ encontram-se pouco ou nada satisfeitos com o tratamento. De referir a possibilidade de existência de um viés de informação, quer pelo facto dos participantes corresponderem aos utentes que se deslocaram à USF por diferentes motivos (entre os quais poderá ter estado a dor não controlada) quer pelo facto dos questionários serem de autopreenchimento pelos utentes, o que pode ter condicionado erros de interpretação e, consequentemente, de preenchimento das respostas. No entanto, entende-se que os resultados encontrados são de extrema importância, pelo que urge intervir mais eficazmente no controlo da dor.

Relativamente às características sociodemográficas, os indivíduos do género feminino, divorciados e viúvos, com escolaridade até nove anos, reformados e reformados por invalidez estão em proporção superior no grupo da omalgia crónica. Pode-se inferir que, em grande parte dos casos, a baixa escolaridade pode relacionar-se com profissões com maior exigência de força física, o que poderá justificar a sua associação com o quadro de dor. No entanto, não é possível inferir esta associação, uma vez que não foram estudadas as profissões dos indivíduos. As evidências disponíveis apenas dão conta de uma associação entre profissões fisicamente mais exigentes e incapacidade funcional. ${ }^{18}$

No grupo de doentes com omalgia crónica verificou-se uma prevalência de ansiedade superior à do grupo sem dor. De um modo semelhante, a prevalência de depressão foi cerca de duas vezes superior nos doentes com omalgia crónica relativamente ao grupo de indivíduos sem dor. Estudos anteriores mostram que a depressão pode ser consequência da dor crónica ${ }^{19}$ por desencadear um sentimento de frustração e impotência nas atividades de vida diária. Contudo, também a perceção de dor pode ser exacerbada por psicopatologia como a ansiedade e a depressão, que constituem em si fatores predisponentes independentes de dor crónica. ${ }^{3,20-21}$ Neste sentido, ao nível da medicina geral e familiar, torna-se fundamental a aplicação do modelo de abordagem biopsicossocial, no qual subjaz o princípio de que a experiência da dor é determinada pela interação dinâmica entre fatores biológicos, psicológicos e sociais. ${ }^{22}$

Relativamente à análise da incapacidade funcional nos doentes com omalgia crónica, a pontuação média de incapacidade foi superior nos indivíduos com ansiedade e presença conjunta de depressão e ansiedade, género feminino, viúvos e reformados, com significado estatístico, tendo em conta a população do estudo. Está estabelecido que a ansiedade, a depressão e os problemas músculo-esqueléticos estão entre as principais causas de anos vividos com incapacidade. ${ }^{23-24} \mathrm{Com}$ parando com o estudo da dor crónica a nível nacional, ${ }^{2}$ apenas o género feminino foi um fator comum de associação com incapacidade. À exceção do estado civil, o estudo EpiReumaPt demonstrou que na população portuguesa os indivíduos com sintomas de ansiedade ou depressão, do sexo feminino e reformados, com patologia músculo-esquelética, também apresentam resultados de incapacidade significativamente superiores. ${ }^{25}$ Pensa-se que estes achados estarão relacionados com uma maior vulnerabilidade destes indivíduos e, 
como tal, valorizam mais as suas limitações e pontuam mais alto na escala HAQ-DI.

Por último, constatou-se que o OR de omalgia crónica aumenta com o grau de incapacidade, a ansiedade, a idade e a baixa escolaridade (inferior ou igual a nove anos). Por comparação com o estudo nacional de dor crónica, que não distingue as variáveis mediante o tipo de localização da dor, apenas a idade e o baixo nível de escolaridade se associam significativamente à dor. ${ }^{2}$ Dever-se-á interpretar com prudência o OR elevado do grau de incapacidade, pois apesar de ser significativo, apenas indica a probabilidade de valores mais elevados do score corresponderem aos doentes com omalgia relativamente aos doentes sem dor. O desenho transversal do estudo é uma limitação que não permite a inferência de causalidade nas variáveis associadas à omalgia crónica e não se pode afirmar se a incapacidade é um preditor de omalgia ou se ocorre o inverso.

\section{CONCLUSÃO}

A prevalência de omalgia crónica foi aproximadamente sete vezes superior à esperada inicialmente. $\mathrm{O}$ estudo permitiu concluir que esta patologia em específico está associada ao grau de incapacidade e à ansiedade, à idade e à baixa escolaridade.

Verificou-se que na maioria dos casos a dor está mal controlada, existindo uma grande insatisfação dos utentes quanto ao tratamento efetuado. A implementação de protocolos clínicos de diagnóstico e atuação na dor crónica, quer dentro das USF quer em conjunto com outras especialidades e/ou valências hospitalares (como a ortopedia, a reumatologia, a medicina física e de reabilitação, a consulta da dor, entre outras), afigura-se bastante importante para o controlo mais eficaz da dor crónica.

\section{AGRADECIMENTOS}

Os autores agradecem ao Dr. Carlos Matos, Dra. Nahida Darwich Sobrino, Dra. Patrícia Marques e Dra. Susana Figueiredo.

\section{REFERÊNCIAS BIBLIOGRÁFICAS}

1. Smith $B H$, Hopton JL, Chambers WA. Chronic pain in primary care. Fam Pract. 1999;16(5):475-82.

2. Azevedo LF, Costa-Pereira A, Mendonça L, Dias CC, Castro-Lopes JM. Epidemiology of chronic pain: a population-based nationwide study on its prevalence, characteristics and associated disability in Portugal. J Pain. 2012;13(8):773-83.
3. Bair MJ, Wu J, Damush TM, Sutherland JM, Kroenke K. Association of depression and anxiety alone and in combination with chronic musculoskeletal pain in primary care patients. Psychosom Med. 2008;70 (8):890-7.

4. Pereira-da-Silva JA. Reumatologia prática. $2^{\mathrm{a}}$ ed. Coimbra: Diagnósteo; 2005. ISBN 9729039267

5. Roh YH, Noh JH, Oh JH, Baek GH, Gong HS. To what degree do shoulder outcome instruments reflect patients' psychologic distress? Clin Orthop Relat Res. 2012;470(12):3470-7.

6. Van den Dolder P, Ferreira P, Refshauge K. Is soft tissue massage an effective treatment for mechanical shoulder pain? A study protocol. J Man Manip Ther. 2010;18(1):50-4.

7. Van der Windt DA, Koes BW, de Jong BA, Bouter LM. Shoulder disorders in general practice: incidence, patient characteristics, and management. Ann Rheum Dis. 1995;54(12):959-64.

8. International Association for the Study of Pain, Subcommittee on Taxonomy. Classification of chronic pain: descriptions of chronic pain syndromes and definitions of pain terms. Pain Suppl. 1986;3:S1-226.

9. Direção-Geral da Saúde.A dor como $5^{\circ}$ sinal vital - Registo sistemático da intensidade da dor: circular normativa n 9/DGCG, de 14/06/2003. Lisboa: DGS; 2003.

10. Fries JF, Spitz P, Kraines RG, Holman HR. Measurement of patient outcome in arthritis. Arthritis Rheum. 1980;23(2):137-45.

11. Fries JF, Spitz PW, Young DY. The dimensions of health outcomes: the Health Assessment Questionnaire Disability and Pain Scales.J Rheumatol. 1982;9(5):789-93.

12. Santos RA, Reis P, Rebelo L, Dias FC, Rosa CM, Queiroz MV. Health Assessment Questionnaire (versão curta): adaptação para língua portuguesa e estudo da sua aplicabilidade ['Health Assessment Questionnaire' adaptation for the portuguese language and evaluation of its applicability]. Acta Reum Port. 1996;XXI(76):15-20. Portuguese

13. Bruce B, Fries JF. The Stanford Health Assessment Questionnaire: dimensions and practical applications. Health Qual Life Outcomes. 2003; 1:20.

14. Pais-Ribeiro J, Silva I, Ferreira T, Martins A, Meneses R, Baltar M. Validation study of a Portuguese version of the Hospital Anxiety and Depression Scale. Psychol Health Med. 2007;12(2):225-35.

15. Bjelland I, Dahl AA, Haug TT, Neckelmann D. The validity of the Hospital Anxiety and Depression Scale: an updated literature review. J Psychosom Res. 2002;52(2):69-77.

16. Snaith RP. The Hospital Anxiety and Depression Scale. Health Qual Life Outcomes. 2003;1:29.

17. Reid KJ, Harker J, Bala MM, Truyers C, Kellen E, Bekkering GE, et al. Epidemiology of chronic non-cancer pain in Europe: narrative review of prevalence, pain treatments and pain impact. Curr Med Res Opin. 2011;27(2):449-62.

18. Beltrán-Sánchez H, Pebley A, Goldman N. Links between primary occupation and functional limitations among older adults in Mexico. SSM Popul Health. 2017;3:382-92.

19. Breivik H, Collett B, Ventafridda V, Cohen R, Gallacher D. Survey of chronic pain in Europe: prevalence, impact on daily life, and treatment. Eur J Pain. 2006;10(4):287-333.

20. Bair MJ, Robinson RL, Katon W, Kroenke K. Depression and pain comorbidity: a literature review. Arch Intern Med. 2003;163(20):2433-45. 
21. McWilliams LA, Cox BJ, Enns MW. Mood and anxiety disorders associated with chronic pain: an examination in a nationally representative sample. Pain. 2003;106(1-2):127-33.

22. Asmundson GJ, Wright KD. Biopsychosocial approaches to pain. In: Hadjistavropoulos T, Craig KD, editors. Pain: psychological perspectives. New York: Psychology Press; 2004. p. 35-53. ISBN 9780415650618

23. Institute for Health Metrics and Evaluation. GBD 2019 [homepage] Seattle: IHME; 2020 [updated 2020 Sep 15].Available from: http://www. healthdata.org/portugal? language $=129$

24. GBD 2017 Disease and Injury Incidence and prevalence Collaborators. Global, regional, and national incidence, prevalence, and years lived with disability for 354 diseases and injuries for 195 countries and territories, 1990-2017: a systematic analysis for the Global Burden of Disease Study 2017. Lancet. 2018;392(10159):1789-858.

25. Branco JC, Rodrigues AM, Gouveia N, Eusébio M, Ramiro S, Machado PM, et al. Prevalence of rheumatic and musculoskeletal diseases and their impact on health-related quality of life, physical function and mental health in Portugal: results from EpiReumaPt (a national health survey). RMD Open. 2016;2(1):e000166.

\section{CONFLITO DE INTERESSES \\ Os autores declaram não possuir quaisquer conflitos de interesse.}

\section{FONTES DE FINANCIAMENTO}

Os autores declaram não ter recebido quaisquer subsídios ou bolsas para a elaboração do artigo.

\section{ENDEREÇO PARA CORRESPONDÊNCIA}

Ana Catarina Sá Machado Correio

E-mail: acatmachado@gmail.com

https://orcid.org/0000-0001-8788-2736

Recebido em 07-11-2018

Aceite para publicação em 08-06-2020

\section{ABSTRACT}

\section{CHRONIC OMALGIA AND ASSOCIATION WITH DISABILITY FUNCTIONAL AND ANXIETY/DEPRESSION:THE REALITY OF FIVE FAMILY HEALTHCARE UNITS OF THE METROPOLITAN AREA OF PORTO}

Objectives: To estimate the prevalence of chronic shoulder pain in the population of five Family Healthcare Units, to describe its semiological characteristics and the sociodemographic characteristics of this population. Secondarily, we intended to evaluate and quantify the association between chronic shoulder pain and functional disability, sociodemographic characteristics, and anxiety/depressive disorders.

Type of study: Cross-sectional study.

Location: Five Family Healthcare Units of Porto's Metropolitan Area.

Population: We obtained a sample of 1,718 patients between the ages of 18 and 64 from the population that visited the five Family Healthcare Units.

Methods: We use a questionnaire to collect sociodemographic and chronic shoulder pain variables as well as two validated scales for the Portuguese population: Health Assessment Questionnaire-Disability Index and Hospital Anxiety and Depression Scale. We tested the association between chronic shoulder pain, functional disability, and anxiety/depression using the multinomial logistic regression model.

Results: The estimated prevalence of chronic shoulder pain was $29.6 \%$, confidence interval of $95 \%$ [27.4-31.8]; $57.8 \%$ patients had daily pain, $70.7 \%$ had moderate to severe pain, and $41.1 \%$ had little/no satisfaction with the treatment. It was found an association between pain degree or location and emotional status, gender, marital status, education, retirement, and retirement due to disability $(p<0.001)$. In the chronic shoulder pain group, female gender, divorce, and widowhood, lower education, retirement due to disability, and anxiety/depression, were in a higher proportion. The higher odds ratio for chronic shoulder pain was found with a higher disability degree, anxiety, age, and lower education (nine years or less).

Conclusions: Chronic shoulder pain prevalence was approximately seven times higher than initially expected. We found an association between chronic shoulder pain and the degree of disability and anxiety, which agrees with the reviewed literature. This study shows an urgent need to intervene effectively in the control of chronic shoulder pain.

Keywords: Chronic shoulder pain; Functional disability; Anxiety; Depression. 